\title{
Naturaleza de los indicadores de gestión en las empresas de rehabilitación de pozos
}

\author{
Nature of management indicators in well rehabilitation companies
}

Natureza dos indicadores de gestão em empresas de reabilitação de poços

\author{
Lenín Urdaneta \\ leninesneider86@gmail.com \\ ORCID 0000-0002-8338-9423
}

Universidad del Zulia, Núcleo Costa Oriental del Lago, Zulia-Venezuela

Artículo recibido enero 2021 | Arbitrado en febrero 2021 | Publicado en mayo 2021

RESUMEN

En la investigación se analizó la naturaleza de los indicadores de gestión aplicados en las empresas de rehabilitación de pozos. Se clasificó como descriptiva, con un diseño no experimental, transeccional y de campo. Los datos se recolectaron de las empresas prestatarias del servicio de rehabilitación de pozos a la industria petrolera venezolana en el Lago de Maracaibo. La técnica de recolección de datos fue la encuesta y el instrumento un cuestionario de respuesta con escala dicotómica. Se utilizó el método de juicio de expertos para darle validez al mismo y se aplicó el coeficiente estadístico Küder-Richardson para el cálculo de su confiabilidad, resultando 0,923 . Se concluye que estos tipos de indicadores, aun cuando son una herramienta útil en el proceso de evaluación, existe una baja aplicación de ellos con lo cual se logre reflejar el comportamiento de los signos vitales o factores clave de las empresas analizadas.

Palabras clave: Indicadores de gestión; naturaleza; rehabilitación de pozos

ABSTRACT

RESUMO

The research analyzed the nature of the management indicators applied in the well rehabilitation companies. It was classified as descriptive, with a non-experimental, transectional and field design. The data was collected from companies providing the well rehabilitation service to the Venezuelan oil industry in Lake Maracaibo. The data collection technique was the survey and the instrument a response questionnaire with a dichotomous scale. The expert judgment method was used to validate it and the Küder - Richardson statistical coefficient was applied to calculate its reliability, resulting in 0.923 . It is concluded that these types of indicators, even when they are a useful tool in the evaluation process, there is a low application of them, which is able to reflect the behavior of the vital signs or key factors of the companies analyzed.

Key words: Management indicators; nature; well rehabilitation
A pesquisa analisou a natureza dos indicadores de gestão aplicados nas empresas de reabilitação de poços. Foi classificado como descritivo, com desenho não experimental, transversal e de campo. Os dados foram coletados junto às empresas prestadoras de serviço de reabilitação de poços para a indústria petrolífera venezuelana no Lago Maracaibo. A técnica de coleta de dados foi o survey e o instrumento um questionário de resposta com escala dicotômica. Para sua validação, foi utilizado o método de julgamento de especialistas e aplicado o coeficiente estatístico de Küder - Richardson para calcular sua confiabilidade, resultando em 0,923. Conclui-se que estes tipos de indicadores, mesmo quando se tratam de uma ferramenta útil no processo de avaliação, são pouco aplicados, refletindo assim o comportamento dos sinais vitais ou fatores-chave das empresas analisadas.

Palavras-chave: Indicadores de gestão; natureza; reabilitação de poços 
INTRODUCCIÓN

El cumplimiento de metas y objetivos constituye el pilar fundamental de toda empresa. Para lograrlo, se han diseñado muchas herramientas a fin de ayudar a los gerentes a transformar las empresas que dirigen en organizaciones más competitivas. Como resultado de lo expuesto, se generan indicadores que permitan seguir y controlar los procesos para así tomar acciones y medidas que contribuyen a la mejora continua, desde la identificación de oportunidades de mejoramiento, con base en una toma de decisión efectiva que permita enfocar la atención de los colaboradores hacia la generación de los mejores resultados. Así, en las empresas, los llamados indicadores son valores cuantificados presentados de forma gráfica, que permiten visualizar el comportamiento de diversas variables que se han determinado en las mismas.

Al respecto, Pardo y Lucio (2016) los definen como un valor o una relación de valores registrados, calculados y observados en conjunto, que permiten medir la satisfacción de las necesidades del cliente, como interactúan las diferentes variables en el proceso y de qué manera se están utilizando los recursos involucrados; también se puede decir que un indicador es la expresión matemática que cuantifica el estado de las características o hechos que se quieren controlar.

Aseveran los autores, que para establecer indicadores de gestión efectivos se debe tomar en cuenta lo que se quiere medir, de allí que cada proceso o actividad podrá ser medido de acuerdo al interés de la empresa; por ello, existen diferentes tipos de indicadores, los cuales son clasificados según su naturaleza, para medir rendimientos económicos, eficacia, eficiencia, efectividad, excelencia, productividad o calidad.

Con base a lo expuesto, en las organizaciones, el desarrollo de indicadores de gestión permite tomar mejores decisiones acerca de los procesos, permitiendo aumentar así su capacidad competitiva. Cuando se mide el desempeño, eficiencia y efectividad de los procesos se puede garantizar el cumplimiento de los objetivos planteados en cada uno de los departamentos, requiriéndose un seguimiento permanente, oportuno $y$ periódico de las actividades a desarrollar, para permitir un monitoreo continuo y eficaz de todas las situaciones presentadas, en especial aquellas que les ocasionen pérdidas en tiempo o dinero a las gestiones de la empresa.

Enmarcadas en el contexto referido, se ubican las empresas que prestan servicio de rehabilitación de pozos a la industria petrolera venezolana en el Lago de Maracaibo, las cuales deben facilitar información oportuna y efectiva sobre el comportamiento de sus variables críticas a través de indicadores previamente definidos, para una mejor visualización de sus desviaciones, permitiéndoles formular acciones que faciliten el control operacional de las actividades, cumpliendo así con el proceso de excelencia empresarial adoptado.

Bajo este enfoque, uno de los sectores que debería asumir mayor énfasis en la mejora continua de sus procesos está representado por las empresas prestadoras de servicios a la industria petrolera, en este contexto, en las empresas que brindan servicios de rehabilitación de pozos a la industria petrolera venezolana en el Lago de Maracaibo, es clave la evaluación y mejora continua de la calidad de sus 
servicios, dado el alto nivel de compromiso para con su principal cliente, la industria petrolera.

En este orden de ideas, dichas empresas, se encargan de este proceso, el cual inicia cuando los investigadores identifican un yacimiento de petróleo submarino o en tierra, para luego perforar la roca que permita extraer el petróleo, el mismo requiere una alta inversión de recursos financieros, humanos y técnicos para lograr los objetivos o metas de producción, este proceso como tal incluye la exploración, perforación, cañoneo y completación de pozos.

Debido a la complejidad de sus operaciones, estas empresas manejan diversas variables de alta relevancia para que sus operaciones se lleven a cabo como, que son catalogadas de alta precisión, exigiendo niveles determinantes de control y seguimiento; es por ello que, la incorporación de los indicadores de gestión, como herramienta de evaluación y control, les permite conocer cuáles son los factores que están alterando los procesos, así como establecer las acciones correctivas pertinentes en los procesos de la organización.

En tal sentido, cobró relevancia llevar a cabo una investigación que permitiera analizar la naturaleza de los indicadores de gestión aplicados en estas empresas de rehabilitación de pozos a la industria petrolera venezolana en el Lago de Maracaibo.
MÉTODO

La investigación fue de tipo descriptiva, con diseño no experimental, de campo y transeccional. Se definió la población de estudio, Petróleos de Venezuela, S.A., Terramarine Services, C.A. y Maritime Contractors S.A., donde los sujetos informantes fueron los gerentes, líderes y supervisores mayores, para un total de 22 unidades informantes. Se determinaron como técnica la encuesta y como instrumento de recolección de datos, un cuestionario estructurado, el cual fue sometido al proceso de validación a través de cinco (5) expertos y se determinó la confiabilidad usando el Coeficiente de Küder-Richardson, alcanzando un valor igual a 0,923 .

De seguido, se aplicó el cuestionario a toda la población, luego se analizaron e interpretaron los datos obtenidos a través del análisis estadístico descriptivo, tomando frecuencias absolutas y relativas. En este análisis, se conocieron los indicadores que requerían atención, y que permitieron establecer las reflexiones finales.

\section{RESULTADOS}

La tabla 1 muestra los resultados obtenidos para el indicador: indicadores económicos, correspondiente a la naturaleza de los indicadores de gestión. Los ítems asociados al indicador permiten señalar que, en las organizaciones estudiadas, se tiene baja presencia de los indicadores económicos según el 30,30 \% de los encuestados, signando este aspecto como una debilidad. 
Tabla 1. Indicador: Indicadores económicos

\section{En la empresa donde usted labora:}

1. ¿Determinan el costo realmente incurrido en cada recurso, para luego compararlo con un costo referencial que puede ser un estándar?

2. ¿Miden la capacidad de la empresa para movilizar los recursos financieros en pro del logro de sus objetivos?

3. ¿El costo de los recursos utilizados coincide con el costo establecido en el presupuesto?

\begin{tabular}{|c|c|c|c|c|c|c|c|c|c|c|c|c|}
\hline \multicolumn{8}{|c|}{ ITEMS } & \multicolumn{5}{|c|}{ INDICADOR } \\
\hline $\mathbf{N}^{\circ}$ & $\mathbf{S i}$ & $\%$ & No & $\%$ & $\mathbf{n}$ & \multicolumn{2}{|c|}{ Categoría } & Si & $\%$ & No & $\%$ & $\%$ \\
\hline 34 & 4 & 18,18 & 18 & 81,82 & 22 & MBP & $\mathrm{D}$ & 6,67 & 30,30 & 15,33 & 69,70 & 100 \\
\hline 35 & 13 & 59,09 & 9 & 40,91 & 22 & $\mathrm{AP}$ & $\mathrm{F}$ & & & EGOR & & \\
\hline 36 & 3 & 13,64 & 19 & 86,36 & 22 & MBP & $\mathrm{D}$ & & ja pres & acia / & bilidad & \\
\hline
\end{tabular}

Al desglosar los resultados se evidencia que, en las empresas sujeto a estudio, se da con alta presencia el hecho de que midan la capacidad de la empresa para movilizar los recursos financieros en pro del logro de sus objetivos $(59,09 \%)$, siendo una fortaleza; pero catalogan con muy baja presencia lo inherente a que determinen el costo realmente incurrido en cada recurso para luego compararlo con un costo referencial que puede ser un estándar (18,18\%), y que el costo de los recursos utilizados coincida con el costo establecido en el presupuesto $(13,64 \%)$; siendo debilidades en su medición de indicadores.

Estos resultados, de baja presencia, poseen poca coincidencia entre lo que ocurre en estas empresas y lo señalado por los autores utilizados. De esta manera, no validan lo planteado por Rivero (2015) cuando explica, este tipo de indicador debe utilizarse para determinar el costo realmente incurrido en cada recurso, en cantidad y precio, para posteriormente compararlo con un costo referencial que puede ser un estándar, un estimado, el costo de otro servicio o actuación similar.
Lo evidenciado pone de manifiesto que, en las empresas prestatarias del servicio de rehabilitación de pozos a la industria petrolera venezolana en el Lago de Maracaibo, no se considera medir la proporcionalidad entre los costos incurridos y la medición de una característica o hecho determinado, así como entre los beneficios y relevancia de la decisión soportada con los datos económicos obtenidos.

De acuerdo a los valores de la tabla 2, relacionados con el indicador eficacia, se evidencia que estos indicadores ostentan baja presencia, según la opinión del 40,91 $\%$ de los encuestados, resultando una debilidad en la medición de sus indicadores. Desde el punto de vista de los resultados por ítem, se evidencia como en las empresas analizadas se otorga alta presencia solo a la actividad referida a si comparan el total de metas alcanzadas en relación al total establecidas cuya frecuencia en la opción afirmativa se ubicó en $54,55 \%$; signándola como una fortaleza para estas empresas. 
Tabla 2. Indicador: Indicadores de eficacia

\section{En la empresa donde usted labora:}

4. ¿Comparan el total de metas alcanzadas en relación al total establecidas?

5. ¿Miden el logro de los resultados permitiéndoles conocer si se hicieron los procesos que se debían hacer?

6. ¿La eficacia de sus procesos les ha permitido mantener ventajas comparativas para sostener una posición destacada en el entorno en que actúan?

\begin{tabular}{ccccccccccccc}
\multicolumn{1}{c}{ ITEMS } & \multicolumn{1}{c}{ INDICADOR } \\
$\mathbf{N}^{\circ}$ & $\mathbf{S i}$ & $\mathbf{\%}$ & $\mathbf{N o}$ & $\mathbf{\%}$ & $\mathbf{n}$ & \multicolumn{1}{c}{ Categoría } & $\mathbf{S i}$ & $\mathbf{\%}$ & No & $\mathbf{\%}$ & $\mathbf{\%}$ \\
37 & 12 & 54,55 & 10 & 45,45 & 22 & AP & D & $\mathbf{9 , 0 0}$ & $\mathbf{4 0 , 9 1}$ & $\mathbf{1 3 , 0 0}$ & $\mathbf{5 9 , 0 9}$ & $\mathbf{1 0 0}$ \\
38 & 9 & 40,91 & 13 & 59,09 & 22 & BP & F & \multicolumn{4}{c}{ CATEGORÍA } \\
39 & 6 & 27,27 & 16 & 72,73 & 22 & MBP & D & \multicolumn{3}{c}{ Baja presencia / Debilidad } \\
\hline
\end{tabular}

Sin embargo, categorizaron con baja presencia lo concerniente a que midan el logro de los resultados permitiéndoles conocer si se hicieron los procesos que se debían hacer (40,91\%); y con muy baja presencia lo referente a si la eficacia de sus procesos les ha permitido mantener ventajas comparativas para sostener una posición destacada en el entorno en que actúan $(27,27 \%)$, declarándolas como debilidades.

Las evidencias anteriores indican poca congruencia con los postulados de Salguero (2015) cuando asevera, los indicadores de eficacia deben ser utilizados para volver los insumos más productivos en el logro de los objetivos, dado que ellos refieren el grado de cumplimiento de los objetivos planteados, en qué medida la organización como un todo, o un área específica de esta, está cumpliendo con sus objetivos estratégicos, sin considerar específicamente los recursos asignados para ello.

Las evidencias encontradas permiten afirmar que, en las empresas prestatarias del servicio de rehabilitación de pozos a la industria petrolera venezolana en el Lago de Maracaibo, los indicadores de eficacia no están siendo aplicados para medir el logro de los objetivos propuestos sin tomar en cuenta los recursos empleados, es decir, para medir el grado en que se alcanzaron los objetivos o metas de la organización. En efecto dada su baja presencia, se puede decir que estas empresas no están midiendo la eficacia pues en los indicadores no se han expresado de manera explícita los criterios de actuación, aclarando que la capacidad de actuar debe estar en concordancia con los criterios previstos, y se mide en términos de porcentaje de cumplimiento del criterio.

Con relación al indicador eficiencia, los valores de respuestas obtenidos en la tabla 3 indican que el 37,88 \% de los encuestados consideran afirmativo lo relativo a que se da baja presencia al uso de estos indicadores como parte de los indicadores de gestión utilizados, confiriéndole una debilidad a las empresas en este sentido.

$\mathrm{Al}$ observar al detalle los resultados, se evidencia que los tres ítems comprendidos en este indicador fueron catalogados con la categoría baja presencia, cuyas frecuencias son $36,36 \%, 40,91 \%$ y $36,36 \%$ al consultarles si utilizan indicadores para 
medir los recursos empleados en el proceso productivo, miden la relación entre lo que se produce y los recursos que se consumen, y consideran la eficiencia de la producción como el proceso de alcanzar los mayores resultados económicos con los menores gastos, respectivamente; catalogando estas actividades como debilidades en la medición de sus indicadores.

Tabla 3. Indicador: Indicadores de eficiencia

\section{En la empresa donde usted labora:}

7. ¿Utilizan indicadores para medir los recursos empleados en el proceso productivo?

8. ¿Miden la relación entre lo que se produce y los recursos que se consumen?

9. ¿Consideran la eficiencia de la producción como el proceso de alcanzar los mayores resultados económicos con los menores gastos?

\begin{tabular}{|c|c|c|c|c|c|c|c|c|c|c|c|c|}
\hline \multicolumn{8}{|c|}{ ITEMS } & \multicolumn{5}{|c|}{ INDICADOR } \\
\hline $\mathbf{N}^{\circ}$ & $\mathbf{S i}$ & $\%$ & No & $\%$ & $\mathbf{n}$ & Cat & & $\mathbf{S i}$ & $\%$ & No & $\%$ & $\%$ \\
\hline 40 & 8 & 36,36 & 14 & 63,64 & 22 & BP & D & 8,33 & 37,88 & 13,67 & 62,12 & 100 \\
\hline 41 & 9 & 40,91 & 13 & 59,09 & 22 & $\mathrm{BP}$ & D & & & EGOR & & \\
\hline 42 & 8 & 36,36 & 14 & 63,64 & 22 & $\mathrm{BP}$ & D & & jja pres & acia / 1 & bilidad & \\
\hline
\end{tabular}

Los referidos señalamientos no validan lo planteado por Salguero (2015) cuando expone, estos indicadores deben ser considerados relevantes para medir el desempeño de toda gestión, pues evalúan la relación entre los recursos y el grado de aprovechamiento por parte de los procesos o actividades del sistema, o sea cumplimiento de la programación establecida y nivel de desperdicio, entre otros.

Sobre la base de lo observado, en las empresas prestatarias del servicio de rehabilitación de pozos a la industria petrolera venezolana en el Lago de Maracaibo, no están aplicando adecuadamente estos indicadores, obviando que los mismos permiten medir la eficiencia, lo cual les permitiría ir más allá de la consecución de los objetivos y metas, ampliando la planificación de gestión hacia la optimización de los recursos disponibles para lograr la mayor cantidad de bienes y servicios posibles; ello facilitará el ser más competitivos, aprovechar mejor de lo que se dispone $y$, por ende, ser mejor empresa.

Los valores mostrados en la tabla 4 revelan que el 33,33 \% de los sujetos encuestados consideran que las actividades relacionadas a la medición de los indicadores de efectividad se realizan en las empresas analizadas con baja presencia, siendo esto una debilidad para su medición de indicadores.

Así, otorgan baja presencia al hecho de que posean indicadores de gestión para medir la relación entre lo realizado y la necesidad del servicio (31,82\%); utilicen indicadores de gestión para medir en qué grado la actividad cumplida satisface la demanda del servicio en términos generales $(40,91 \%) ; y$ que los indicadores de gestión aplicados involucren el logro de los resultados programados en el tiempo a los costos más razonables posibles $(27,27 \%)$, distinguiendo estos aspectos como debilidades. 
Tabla 4. Indicador: Indicadores de efectividad

\section{En la empresa donde usted labora:}

10. ¿Poseen indicadores de gestión para medir la relación entre lo realizado y la necesidad del servicio?

11. ¿Utilizan indicadores de gestión para medir en qué grado la actividad cumplida satisface la demanda del servicio en términos generales?

12. ¿Los indicadores de gestión implementados involucran el logro de los resultados programados en el tiempo a los costos más razonables posibles?

\begin{tabular}{|c|c|c|c|c|c|c|c|c|c|c|c|c|}
\hline \multicolumn{8}{|c|}{ ITEMS } & \multicolumn{5}{|c|}{ INDICADOR } \\
\hline $\mathbf{N}^{\circ}$ & $\mathbf{S i}$ & $\%$ & No & $\%$ & $\mathbf{n}$ & Cat & & $\mathbf{S i}$ & $\%$ & No & $\%$ & $\%$ \\
\hline 43 & 7 & 31,82 & 15 & 68,18 & 22 & $\mathrm{BP}$ & $\mathrm{D}$ & 7,33 & 33,33 & 14,67 & 66,67 & 100 \\
\hline 44 & 9 & 40,91 & 13 & 59,09 & 22 & $\mathrm{BP}$ & $\mathrm{D}$ & & & [EGOR & & \\
\hline 45 & 6 & 27,27 & 16 & 72,73 & 22 & $\mathrm{BP}$ & $\mathrm{D}$ & & jja pre & ncia / I & ebilidad & \\
\hline
\end{tabular}

Las afirmaciones precedentes no alcanzan a validar lo postulado por Salguero (2015) cuando asevera, las organizaciones pudieran medir beneficios de carácter económico, como tasa de rentabilidad, verificando que, al menos, los gastos generados estén equilibrados con relación a los ingresos; de este modo, la efectividad mide el impacto final de la gestión de una organización sobre la población afectada, en donde el valor potencial creado por la empresa no se mide únicamente en el bien o servicio, sino en relación con los resultados e impacto que genera en la población.

Por lo evidenciado, en las empresas prestatarias del servicio de rehabilitación de pozos a la industria petrolera venezolana en el Lago de Maracaibo, no están considerando una buena aplicación de los indicadores de efectividad, dejando de reconocer que estos les permitirían medir el impacto de la gestión, tanto en el logro de los resultados como en el manejo de los recursos utilizados y disponibles, considerando que, estos indicadores son el resultado de la eficacia y la eficiencia.

Seguidamente, se presentan en la tabla 5 los resultados para el indicador: indicadores de excelencia. Se observa una concentración de respuestas afirmativas del $21,21 \%$, otorgándole la categoría de muy baja presencia, según el baremo utilizado, indicando debilidades para las empresas en la medición de sus indicadores de desempeño. 
Tabla 5. Indicador: Indicadores de excelencia

En la empresa donde usted labora:

13. ¿Tienen establecidos mecanismos para medir la satisfacción de los requerimientos de los clientes?

14. ¿Analizan la información recolectada sobre la satisfacción de los clientes para acentuar lo que se está haciendo bien?

15. ¿Analizan la información recolectada sobre la satisfacción de los clientes para corregir aquello que está generando insatisfacción en los mismos?

\begin{tabular}{|c|c|c|c|c|c|c|c|c|c|c|c|c|}
\hline \multicolumn{8}{|c|}{ ITEMS } & \multicolumn{5}{|c|}{ INDICADOR } \\
\hline $\mathbf{N}^{\circ}$ & $\mathbf{S i}$ & $\%$ & No & $\%$ & $\mathbf{n}$ & \multicolumn{2}{|c|}{ Categoría } & $\mathbf{S i}$ & $\%$ & No & $\%$ & $\%$ \\
\hline 46 & 4 & 18,18 & 18 & 81,82 & 22 & MBP & $\mathrm{D}$ & 4,67 & 21,21 & 17,33 & 78,79 & 100 \\
\hline 47 & 5 & 22,73 & 17 & 77,27 & 22 & MBP & $\mathrm{D}$ & \multicolumn{5}{|c|}{ CATEGORÍA } \\
\hline 48 & 5 & 22,73 & 17 & 77,27 & 22 & MBP & $\mathrm{D}$ & \multicolumn{5}{|c|}{ Muy baja presencia / Debilidad } \\
\hline
\end{tabular}

De esta manera, al consultarles si tienen establecidos mecanismos para medir la satisfacción de los requerimientos de los clientes; analizan la información recolectada sobre la satisfacción de los clientes para acentuar lo que se está haciendo bien y para corregir aquello que está generando insatisfacción en los mismos; el $18,18 \%$; $22,73 \%$ y el $22,73 \%$ respondieron de manera afirmativa, respectivamente; otorgando muy baja presencia a estas actividades, por ende, son debilidades, consideradas oportunidades de mejora según el criterio del investigador.

De acuerdo a los resultados, no se logra congruencia con Salguero (2015), quien asevera que, es fundamental revisar si la gerencia conoce los requerimientos de los clientes y cuáles son estos a nivel de cada proceso específico, de allí la importancia de medir los indicadores de excelencia, pues al considerar un sistema de indicadores de gestión es esencial proponer un mecanismo para medir la satisfacción de los requerimientos de los clientes.

De conformidad con estos resultados, en las empresas prestatarias del servicio de rehabilitación de pozos a la industria petrolera venezolana en el Lago de Maracaibo, no se da mayor importancia a la medición de los indicadores de excelencia, obviando, tal vez, que la excelencia no es más que la calidad del servicio prestado al cliente, reportando calidad y capacidad técnica y resolutiva del servicio. Por ello, no están asumiendo que la medición de estos indicadores reporta la satisfacción de sus clientes, requiriendo la utilización de un enfoque metodológico para no caer en subjetividades $y$ tomar acciones no adecuadas.

La tabla 6 muestra la frecuencia relativa de respuestas correspondientes al indicador designado como indicadores de productividad, el cual posee baja presencia en el desarrollo de las actividades de medición que realizan las empresas analizadas, según el 36,86 \% de los encuestados, viéndolas como debilidades en la medición de sus indicadores. Estos resultados obedecen a los obtenidos para cada uno de los ítems medidos, expresados con alta, baja y muy baja presencia. 
Tabla 6. Indicador: Indicadores de productividad

En la empresa donde usted labora:

16. ¿Utilizan indicadores para medir la relación existente entre la productividad y los recursos empleados?

17. ¿Los indicadores utilizados les permiten detectar fuentes de improductividad generadas por el desperdicio de recursos?

18. ¿Asocian los resultados sobresalientes de su personal con incentivos o bonos productivos?

\begin{tabular}{rrccccccccccc}
\multicolumn{1}{c}{} & \multicolumn{1}{c}{ ITEMS } & \multicolumn{1}{c}{ INDICADOR } \\
$\mathbf{N}^{\circ}$ & $\mathbf{S i}$ & $\mathbf{\%}$ & $\mathbf{N o}$ & $\mathbf{\%}$ & $\mathbf{n}$ & \multicolumn{2}{c}{ Categoría } & Si & $\mathbf{\%}$ & No & $\mathbf{\%}$ & $\mathbf{\%}$ \\
49 & 5 & 22,73 & 17 & 77,27 & 22 & MBP & D & $\mathbf{8 , 0 0}$ & $\mathbf{3 6 , 3 6}$ & $\mathbf{1 4 , 0 0}$ & $\mathbf{6 3 , 6 4}$ & $\mathbf{1 0 0}$ \\
50 & 7 & 31,82 & 15 & 68,18 & 22 & BP & D & \multicolumn{4}{c}{ CATEGORÍA } \\
51 & 12 & 54,55 & 10 & 45,45 & 22 & AP & F & \multicolumn{3}{c}{ Baja presencia / Debilidad } \\
\hline
\end{tabular}

Al analizar detalladamente los resultados obtenidos para cada uno de los ítems del indicador, pudo visualizarse lo siguiente: otorgan alta presencia en lo relativo a que asocien los resultados sobresalientes de su personal con incentivos o bonos productivos (54,55 \%), siendo esto una fortaleza. Mientras, consideran con baja presencia lo correspondiente a que los indicadores utilizados les permiten detectar fuentes de improductividad generadas por el desperdicio de recursos (31,82\%); y con muy baja presencia catalogaron lo tocante a que utilicen indicadores para medir la relación existente entre la productividad y recursos empleados $(22,73 \%)$, calificando estos aspectos como debilidades de las empresas analizadas.

En función del análisis efectuado, se comprueba que existe poca coincidencia con Mora (2016) cuando afirma, el objetivo de un buen administrador debe ser llevar el control de la productividad del proceso, detectar fuentes de improductividad que normalmente son generadas por el desperdicio de recursos o una organización no óptima, se puede decir, además que, para cumplir con este objetivo, se debe llegar al detalle de las actividades del proceso productivo (índices de productividad por actividades).

Los hallazgos encontrados ponen de manifiesto que, en las empresas prestatarias del servicio de rehabilitación de pozos a la industria petrolera venezolana en el Lago de Maracaibo, los indicadores de productividad no están enfocados en el control de la productividad de los procesos, pues se manejan con baja presencia, lo cual no les debe permitir detectar fuentes de improductividad que normalmente son generadas por el desperdicio de recursos o porque las organizaciones no sean óptimas.

Seguidamente, la tabla 7 recoge el comportamiento obtenido para el indicador: indicadores de calidad. En la misma se observa que, en promedio, el $18,18 \%$ de la población encuestada afirma que los indicadores de calidad tienen muy baja presencia como parte de los tipos de indicadores de gestión, según su naturaleza, que aplican, viéndolo como una debilidad de su proceso de medición de indicadores. 
Tabla 7. Indicador: Indicadores de calidad

En la empresa donde usted labora:

19. ¿Evalúan la calidad de los procesos para asegurar la satisfacción de los clientes?

20. ¿Evalúan la calidad de los servicios prestados para asegurar la satisfacción de los clientes?

21. ¿Sus indicadores de calidad orientan las actividades de mejora, mediante la implantación de acciones preventivas?

\begin{tabular}{|c|c|c|c|c|c|c|c|c|c|c|c|c|}
\hline \multicolumn{8}{|c|}{ ITEMS } & \multicolumn{5}{|c|}{ INDICADOR } \\
\hline $\mathbf{N}^{\circ}$ & $\mathbf{S i}$ & $\%$ & No & $\%$ & $\mathbf{n}$ & \multicolumn{2}{|c|}{ Categoría } & Si & $\%$ & No & $\%$ & $\%$ \\
\hline 52 & 4 & 18,18 & 18 & 81,82 & 22 & MBP & $\mathrm{D}$ & 4,00 & 18,18 & 18,00 & 81,82 & 100 \\
\hline 53 & 4 & 18,18 & 18 & 81,82 & 22 & MBP & $\mathrm{D}$ & & & [EGOR & & \\
\hline 54 & 4 & 18,18 & 18 & 81,82 & 22 & MBP & $\mathrm{F}$ & & baja pr & sencia & Debilic & \\
\hline
\end{tabular}

Se observa que, el 18,18 \% de los encuestados están de acuerdo en que evalúan la calidad de los procesos para asegurar satisfacción de los clientes; evalúan la calidad de los servicios prestados para asegurar la satisfacción de los clientes; y sus indicadores de calidad orientan las actividades de mejora, mediante la implantación de acciones preventivas; confiriéndole muy baja presencia a estas actividades en la medición de los indicadores de calidad que se miden, siendo esto atribuible a debilidades.

Puede decirse que no se validan los postulados de Mora (2016) cuando explica, un indicador de calidad puede orientar las actividades de mejora, mediante la implantación de acciones preventivas y control de los resultados obtenidos; al tiempo que permite tener bajo control el proceso, de manera que, si supera los límites admisibles, se tomen las medidas correctoras y preventivas correspondientes.

Lo mostrado anteriormente, permite evidenciar que, en las empresas prestatarias del servicio de rehabilitación de pozos a la industria petrolera venezolana en el Lago de Maracaibo, los indicadores de calidad no son asumidos como un instrumento de medición basado en hechos y datos que les permita evaluar la calidad de sus procesos, productos y servicios para asegurar satisfacción en determinada actividad o proceso empresarial. Al mismo tiempo, no están considerando que estos indicadores influyen sobre los indicadores de gestión de toda la organización, los cuales debe utilizar la dirección para proponer acciones globales o para controlar determinados parámetros del negocio.

Finalmente, como se aprecia en la tabla 8 , el resumen para la naturaleza de los indicadores de gestión, la cual muestra un valor de 31,17 \% de respuestas afirmativas, reflejando, de acuerdo a la opinión de los encuestados, muy baja presencia de los tipos de indicadores de gestión según su naturaleza en las empresas prestatarias del servicio de rehabilitación de pozos a la industria petrolera venezolana en el Lago de Maracaibo. 
Tabla 8. Naturaleza de los indicadores de gestión

\begin{tabular}{|c|c|c|c|c|c|}
\hline INDICADOR & Si & $\%$ & No & $\%$ & BAREMO \\
\hline Indicadores económicos & 6,67 & 30,30 & 15,33 & 69,70 & $\begin{array}{l}\text { Baja presencia } \\
\text { Debilidad } \\
\text { Baja presencia }\end{array}$ \\
\hline Indicadores de eficacia & 9,00 & 40,91 & 13,00 & 59,09 & $\begin{array}{c}\text { Debilidad } \\
\text { Baja presencia }\end{array}$ \\
\hline Indicadores de eficiencia & 8,33 & 37,88 & 13,67 & 62,12 & $\begin{array}{c}\text { Debilidad } \\
\text { Baja presencia }\end{array}$ \\
\hline Indicadores de efectividad & 7,33 & 33,33 & 14,67 & 66,67 & $\begin{array}{c}\text { Debilidad } \\
\text { Muy baja presencia }\end{array}$ \\
\hline Indicadores de excelencia & 4,67 & 21,21 & 17,33 & 78,79 & $\begin{array}{c}\text { Debilidad } \\
\text { Baja presencia }\end{array}$ \\
\hline Indicadores de productividad & 8,00 & 36,36 & 14,00 & 63,64 & $\begin{array}{c}\text { Debilidad } \\
\text { Muy baja presencia }\end{array}$ \\
\hline Indicadores de calidad & 4,00 & 18,18 & 18,00 & 81,82 & Debilidad \\
\hline Totales & 6,86 & 31,17 & 15,14 & 68,83 & $\begin{array}{c}\text { Baja presencia } \\
\text { Debilidad }\end{array}$ \\
\hline
\end{tabular}

Adicionalmente, se observa que los tipos de indicadores: económicos, de eficacia, de eficiencia, de efectividad y de productividad muestran baja presencia en la medición de sus indicadores de gestión; mientras, los indicadores de excelencia y de calidad poseen muy baja presencia; siendo todos considerados como debilidades de las empresas en su proceso de medición del desempeño.

Estos resultados, de baja presencia, permiten visualizar poca coincidencia con la teoría manejada, en lo que respecta a lo planteado por Rivero (2015) cuando plantea, los indicadores de gestión se clasifican según su naturaleza con base a los factores claves de éxito; definitivamente, los indicadores de gestión deben reflejar el comportamiento de los signos vitales o factores clave (algunos autores los llaman factores críticos). Contar con un conjunto de indicadores, en el proceso de medición del desempeño de la empresa, que abarque los factores clave, implica garantizar la integridad de la función de apoyo para la toma de decisiones.
De igual forma, estos resultados no validan a Delgado (2015) al aseverar que, los indicadores de gestión pueden clasificarse según su naturaleza, en función del componente de la actuación que tratan de medir. Tampoco son congruentes con lo señalado por Mora (2016) cuando establece, los indicadores pueden clasificarse según la naturaleza del objeto a medir, donde se pueden distinguir los siguientes tipos: económicos, eficacia, eficiencia, efectividad, excelencia, productividad y de calidad.

De acuerdo a lo evidenciado, en las empresas prestatarias del servicio de rehabilitación de pozos, no se está considerando que después de haber establecido los objetivos y conocida la capacidad de gestión, el paso siguiente debe ser establecer los indicadores que sirvan de apoyo y guía para cada miembro y equipo de la organización. Así entonces, los tipos de indicadores a asumir deben medir las actividades realizadas, recursos utilizados y resultados obtenidos; por lo cual, se han detectado oportunidades de mejora en cada uno de los indicadores estudiados. 
CONCLUSIONES

Se pudo detectar el uso de cada tipo de indicador con baja aplicación; no obstante, se evidenció la existencia de ciertas fortalezas, a saber.

En relación a los indicadores económicos, miden la capacidad de la empresa para movilizar los recursos financieros en pro del logro de sus objetivos; en cuanto a los indicadores de eficacia, comparan el total de metas alcanzadas en relación al total de las metas establecidas; para los indicadores de productividad, asocian los resultados sobresalientes de su personal con incentivos 0 bonos productivos.

En términos generales, se concluye que estos tipos de indicadores, aun cuando son una herramienta útil en el proceso de evaluación, en la presente investigación existe una baja aplicación de los mismos y logra reflejar el comportamiento de los signos vitales o factores clave en las empresas analizadas.

\section{REFERENCIAS}

Delgado, J. (2015). Planificando Estratégicamente. Windmills International Editions, Inc. California. USA

Mora, L. (2016). Indicadores de la gestión logística. 2da Edición. Ediciones ECOE. Bogotá. Colombia

Pardo, D. y Lucio, C. (2016). Análisis de indicadores de ciencia y tecnología. Editoras Académicas. Universidad del Rosario. Colombia

Rivero, C. (2015). Indicadores integrales de Gestión. 1era edición. McGraw-Hill. Bogotá Colombia

Salguero, F. (2015). Indicadores de gestión y cuadro de mando integral. Ediciones Díaz de Santos, Madrid. España 